\section{Motivadores ao Compartilhamento de Conhecimento Tácito em Organizações Intensivas em Conhecimento}

\author{
Patrícia Fernanda Dorow, Dorzeli Salete Trzeciak e Gregório Jean \\ Varvakis Rados
}

\section{RESUMO}

O objetivo dessa pesquisa é evidenciar os motivadores no compartilhamento do conhecimento tácito, de acordo com a percepção de radiologistas integrantes de grupos de trabalhos de organizações intensivas em conhecimento. É uma pesquisa aplicada, com abordagem qualitativa e, ainda, descritiva. Como procedimento técnico para a sua realização, utilizouse o estudo multicaso, tendo como universo de pesquisa três organizações intensivas em conhecimento de Santa Catarina. Para a coleta de dados utilizou-se a observação e entrevistas, procedendo à análise dos dados, orientada pela análise temática. Os resultados contribuem para o avanço da teoria existente e apontam que o quando o indivíduo percebe um ambiente favorável ao compartilhamento do conhecimento, onde é reconhecido pelo seu conhecimento, ele é motivado a compartilhar, pois identifica oportunidades de aprender ao discutir casos desafiadores com seus colegas por meio de práticas que são efetivas devido às características peculiares dos grupos e da percepção que os mesmos possuem da ação de compartilhar conhecimento tácito. Conclui-se que o feedback direto e práticas apropriadas, como revisão por pares, storytelling, mapa de conhecimentos e mentoring agem na criação de um comportamento recíproco de cooperação e pertencimento, favorecendo o compartilhamento de conhecimento tácito.

Palavras-chave: Conhecimento; Compartilhamento do conhecimento tácito; Aprendizagem organizacional; Motivação.

\section{Motivators to The Sharing of Tactic Knowledge in Intensive Organizations in Knowledge}

\section{ABSTRACT}

The objective of this research is to highlight the motivators for the sharing of tacit knowledge, according to the perception of radiologists belonging to groups of works from intensive organizations in knowledge. It is an applied research, with a qualitative and descriptive approach. As a technical procedure for its accomplishment, it used the multicaso study, having as research universe three intensive organizations in knowledge of Santa Catarina. The observation and interviews were used for the data collection, proceeded by the analysis of the data, guided by the thematic analysis. The results show that intrinsic motivation, the physical environment, the form of interaction and recognition act in the creation of trust are indispensable factors for the sharing of tacit knowledge. It is concluded that direct feedback and appropriate practices, such as peer review, storytelling, knowledge mapping and mentoring act on the creation of a reciprocal cooperation and belonging behavior, which favors the sharing of tacit knowledge.

\section{Keywords: Knowledge; Sharing of tacit knowledge; Organizational} learning; Motivation.

Recebido em: 04/07/2017 Aprovado em: 02/12/2017 Última Modificação: 20/06/2018

Check for updates

Patrícia Fernanda

Dorow (iD),

Universidade Federal de Santa

Catarina, Brasil.

Doutorado em Engenharia e Gestão do

Conhecimento, Universidade Federal

de Santa Catarina, Brasil

patrciadorow@gmail.com

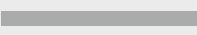

Dorzeli Salete Trzeciak (D), Universidade Federal de Santa Catarina, Brasil.

Pós-Doutorado em Engenharia e Gestão do Conhecimento,

Universidade Federal de Santa Catarina, Brasil

dorzeli@gmail.com

Gregório Jean Varvakis Rados (iD,

Professor Titular da Universidade Federal de Santa Catarina, Depto. de Engenharia do Conhecimento, Brasil. Doutor em Manufacturing Engineering - Loughborough University of

Technology, Inglaterra

g.varvakis@ufsc.br 


\section{Introdução}

O contexto organizacional é importante para organizações intensivas em conhecimento, pois precisam tomar decisões complexas que exigem a articulação de conhecimentos de especialistas de diferentes domínios.

As organizações intensivas em conhecimento, de acordo com Starbuck (1992) se caracterizam pela intensidade de uso de conhecimento no seu domínio de produção. Alvesson (2004) complementa que, em organizações intensivas em conhecimento, a maioria do trabalho é de natureza intelectual, o que exige a resolução complexa de problemas e funcionários bem qualificados constituem a maior parte da força de trabalho. Para esse autor, nesse tipo de organização, a atenção especial é dedicada ao desenvolvimento, compartilhamento e uso do conhecimento, ou seja, o conhecimento é a principal entrada para o desenvolvimento de produtos e/ou serviços.

Sendo assim, esses tipos de organizações dependem muito das pessoas que compõem sua estrutura funcional e da experiência organizacional ou perícia individual que a organização é capaz de desenvolver, com a finalidade de aplicar o conhecimento intensivamente em uma área específica, como é o caso das organizações de radiologia e diagnóstico por imagem. Nesse tipo de organização, os trabalhadores do conhecimento, como mencionam Işik, Mertens e Van Den Bergh (2013), na maioria das vezes, são especialistas com alto nível de conhecimento que demandam autonomia e liberdade para desenvolver seu trabalho.

Stewart (2002) menciona que há várias características que auxiliam a classificação de uma organização como intensiva em conhecimento, dentre elas, está o potencial para compartilhar conhecimento. Ao resolverem problemas complexos não estruturados, as pessoas trazem conhecimento e experiência para a situação em questão e, ao interagir nesse processo, criam, usam e compartilham conhecimento.

Essas interações ocorrem num contexto (ambiente), que se transforma durante as ações de interpretação, que acontecem quando os indivíduos trazem sua experiência sob a forma de conhecimento tácito para a situação que está se desenvolvendo. Esses ambientes ou contextos compartilhados, em que ocorrem essas interações, sejam elas em espaços físicos, virtuais ou mentais, são chamados de "ba" e servem de base para o processo de criação do conhecimento organizacional (Senoo, Magnier-Watanabe, \& Salmador, 2007). Sendo assim, o compartilhamento do conhecimento tácito para a resolução de problemas complexos demanda o surgimento e a manutenção de um contexto compartilhado, ou seja, a capacidade de compreender o surgimento e a transformação do contexto.

A relação entre contexto e compartilhamento de conhecimento tácito é estratégica para o sucesso das organizações, na medida em que enfrentam um ritmo acelerado das operações em uma economia baseada no conhecimento.

Segundo Kim e Lee (2006) o compartilhamento do conhecimento consiste no ato de compartilhar experiências pessoais relacionadas com o seu trabalho, com outros indivíduos, grupos e parceiros na organização. Esse processo possibilita a aprendizagem devido às trocas de conhecimento, 
vivências e desempenho de tarefas que exigem participação de grupos, e não mais pelo trabalho individual (Celadon, 2005).

Ocompartilhamento deconhecimento incluitanto o conhecimento explícito quanto o conhecimento tácito. Além disso, apesar dos processos e das tecnologias ajudarem o compartilhamento de conhecimento, são os indivíduos que decidem por compartilhar ou não seus conhecimentos. Na visão de Gagné (2009), o compartilhamento de conhecimentos tácitos é um componente essencial da criação de conhecimento e é a etapa mais crítica do processo. Porém, apesar da importância do compartilhamento do conhecimento tácito, este é de difícil externalização.

Por outro lado, Ipê (2003) aponta que uma estrutura ambiental adequada facilita o compartilhamento de conhecimento. Essa estrutura abrange tanto os canais de aprendizado intencional como os ambientes virtuais, quanto os canais de relacionamento e aprendizagem que envolvem as interações face a face. Esse autor ainda se refere à motivação para compartilhar como sendo um dos maiores influenciadores ao compartilhamento do conhecimento. Neste sentido, identificar os motivadores ao compartilhamento de conhecimento tácito contribui para melhorar o fluxo de conhecimento e, consequentemente, para a criação de novo conhecimento.

No entanto, o compartilhamento do conhecimento é um desafio para as organizações, devido a vários motivos. Em primeiro lugar, as pessoas possuem conhecimento tácito, que é definido como aquele conhecimento que não é verbalizado, intuitivo e desarticulado (Polanyi, 1966). Em segundo lugar, o impulsionador do compartilhamento é a construção e o desenvolvimento de uma relação entre fonte e destinatário de conhecimento. Se esses dois atores não tiverem confiança mútua, provavelmente suas contribuições não serão reconhecidas (Wang \& Noe, 2010). Em terceiro lugar, o compartilhamento do conhecimento é um comportamento voluntário. Se as pessoas não confiarem em seu próprio conhecimento, poderão relutar em compartilhá-lo com os outros. Por fim, o conhecimento é, muitas vezes, sinônimo de poder, uma vez que pode ser uma fonte de vantagem competitiva sustentável (Nonaka \& Takeuchi, 1995; Van Den Hooff \& De Ridder, 2004; Dixon, 2002; Lin, Lee, \& Wang, 2009).

O que se observa, é que a literatura apresenta uma quantidade limitada de evidências empíricas sobre estratégias para compartilhar conhecimento tácito (Choi \& Lee, 2002; Ipê, 2003; Wang \& Noe, 2010). Dessa forma, estudos que descrevam essa dinâmica podem contribuir para o progresso da organização, uma vez que compartilhar conhecimento tácito contribui significativamente para melhorar o gerenciamento e a prática do conhecimento (Wei, Zheng, \& Zhang, 2011).

Nesse sentido o propósito deste trabalho é evidenciar os motivadores ao compartilhamento de conhecimento tácito, segundo a percepção de radiologistas pertencentes aos grupos de trabalhos de organizações intensivas em conhecimento.

A principal contribuição do trabalho está na melhor compreensão do que motiva os indivíduos em organizações intensivas em conhecimento a compartilharem seus conhecimentos tácitos para a aprendizagem organizacional, indicando, assim, como os ambientes podem suportar o uso de práticas facilitadoras. 
Para atingir esse objetivo, o presente artigo estrutura-se em: introdução, em que se apresenta o contexto e o objetivo do estudo; referencial teórico, que aborda a temática em estudo; procedimentos metodológicos, com a descrição das etapas adotadas para a realização da pesquisa; apresentação e análise dos motivadores ao compartilhamento de conhecimento tácito nas organizações estudadas; considerações finais da pesquisa e recomendações para trabalhos futuros; e as referências utilizadas.

\section{Referencial teórico}

\section{Conhecimento explícito e conhecimento tácito}

Apesar de muito ter sido escrito sobre o tema conhecimento, o debate filosófico sobre sua natureza e seu significado permanece sem solução, conforme apontam Alavi e Leidner (2001) e Davenport e Prusak (1998). Inúmeros pesquisadores definiram conhecimento de diversas maneiras. Davenport e Prusak (1998) definem o conhecimento como um fluxo contínuo de experiências, valores, contexto, informações e percepções que se tornam um modelo para avaliar e integrar novas experiências e informações. Embora alguns pesquisadores acreditem que o conhecimento não existe fora do indivíduo, outros como De Long e Fahey (2000) julgam que artefatos como aplicativos e software possuem conhecimento, porém, de uma forma explícita.

Para o contexto desse artigo, adota-se a abordagem conexionista de Von Krogh e Roos (1995) ao defenderem que o conhecimento está inserido tanto na cabeça dos indivíduos, quanto em suas interações sociais, no nível organizacional. Em síntese, a palavra conhecimento parece ser indescritivel, mas há um forte consenso de que a fonte primária do conhecimento é de natureza tácita (Nomura, 2002; Von Krogh, Nonaka, \& Rechsteiner, 2012). Esse tipo de conhecimento permite que o indivíduo possa lidar com situações novas e diferentes. Exemplos de conhecimento tácito são habilidades para andar de bicicleta ou mesmo reconhecer um rosto.

Conforme Polanyi (1966, p. 4) "sabemos mais do que podemos expressar", ou seja, o conhecimento tácito não pode ser completamente declarado, além de ser ligado a um contexto e estar fortemente relacionado às experiências. Ainda segundo esse autor, o uso da linguagem é fundamental para compartilhar conhecimento, porém, quanto maior a tacitividade do conhecimento, maiores serão as dificuldades para o seu compartilhamento.

Nonaka e Takeuchi (1997) propuseram duas dimensões para o conhecimento organizacional: tácito e explícito. Para o conhecimento tácito Polanyi (1996, p. 4) deu significado de "crença comprometida" que é contextual, tem caráter e é difícil de expressar. Em contraste ao conhecimento tácito, o conhecimento explícito independe do contexto, é facilmente comunicado e codificado, sendo de fácil externalização. É passível de ser disseminado, reproduzido, utilizado e melhorado em toda a organização (Lee, Gillespie, Mann, \& Wearing, 2010). 
Sendo assim, tem sido amplamente aceito que as diversas dimensões do conhecimento incluem componentes tácitos e explícitos (Hildreth \& Kimble, 2002; Nonaka \& Von Krogh, 2009; Polanyi, 1966). Autores como Al-Hawamdeh (2002) e Nonaka, Byosiere, Borucki e Konno (1994), Collins \& Evans (2010) acreditam que o conhecimento tácito pode ser compartilhado por meio da interação social com os outros, durante o processo de conversão do conhecimento, que ocorre entre conhecimento tácito e explícito. Essa tradução, de acordo com Bejinaru e lordache (2010), pode ser auxiliada por meio de metáforas, analogias, descrição, gestos e linguagem corporal.

\section{Motivadores ao compartilhamento do conhecimento tácito}

Segundo Yang e Farn (2009), o conhecimento tácito é individual, sendo assim, não se agrega facilmente ao conhecimento organizacional. O conhecimento tácito está na dimensão cognitiva do indivíduo (Borges, 2012).

Na visão de Ipê (2003) o compartilhamento de conhecimento consiste no ato de tornar o conhecimento disponível para outros dentro de uma organização e denota um processo de aprendizagem. Contudo, os esforços desenvolvidos para otimizar as atividades de compartilhamento do conhecimento são um fenômeno relativamente novo (Husted \& Michailova, 2002).

O compartilhamento do conhecimento em organizações intensivas em conhecimento envolve atividades mútuas e proximidade entre os membros (Benson \& Debroux, 2013). Dessa forma, otimizar e, posteriormente, alavancar esses comportamentos pode ser crítico, uma vez que o ato de compartilhar é voluntário (Mahmood, Qureshi, \& Shahbaz, 2011).

Como já abordado, os indivíduos desempenham um papel crucial na criação de conhecimento, porque ele é criado por meio de suas interações em seu ambiente. Face a isto, o conhecimento é criado primeiramente em forma tácita e, em seguida, explicado aos outros indivíduos em um "ba" condutivo que os motiva a fazê-lo (Stenmark, 2000; Lang, 2001; Hunter, Beaumont, \& Lee, 2002).

Greenberg e Baron (1997, p. 2142) definem a motivação como "o conjunto de processos que despertam, dirigem e mantêm o comportamento humano para alcançar algum objetivo". Dessa forma, é fato que o conhecimento não se move sem força motivadora, e as pessoas não dão bens valiosos, como o conhecimento, sem se preocupar com os ganhos e perdas envolvidas no processo (Davenport \& Prusak, 1998; Stenmark, 2000).

Segundo Sharkie (2005), com confiança, mesmo indivíduos com laços fracos são capazes de compartilhar conhecimento de forma eficaz. A falta de confiança pode ser um inibidor significativo ao compartilhamento do conhecimento. Os indivíduos temem que a externalização do seu conhecimento possa levar à automatização, não sendo mais relevante sua existência na organização (Stenmark, 2000).

Portanto, confiança e lealdade são características raras no local de trabalho (Latham \& Pinder, 2005) e identificar os motivadores ao 
compartilhamento de conhecimento tácito pode ajudar a influenciar a motivação dos indivíduos.

\section{Contexto compartilhado "ba"}

Como afirma Dabbagh e Kitsantas (2012) a aprendizagem representa o conhecimento que é mobilizado por meio da interação social. Da mesma forma, a aprendizagem organizacional realiza a gestão da criação do conhecimento organizacional por meio da interação social estratégica de seus membros (Aizpurúa, Saldaña \& Saldaña, 2011).

O fato é que a aprendizagem organizacional envolve processos, que sustentam ou expandem o desempenho por meio da experiência (Kumaraswamy \& Chitale, 2012).

A falta de um contexto compartilhado de interação social que permite o compartilhamento de conhecimentos, experiências e reflexão entre os membros do grupo, pode dificultar a implementação de estratégias de gestão do conhecimento (De Long \& Fahey, 2000). Segundo Detienne, Dyer, Hoopes e Harris (2004) esse contexto deve envolver: pessoas, liderança, processos e tecnologias.

Para Von Krogh, Ichijo e Nonaka (2001), um espaço compartilhado, que se caracteriza por uma rede de interações e um ambiente adequado para criar conhecimento onde ocorre a criação de uma cultura compartilhada de confiança, estima mútua empatia. Este ambiente é chamado de "ba" e é importante para reunir e compartilhar, principalmente, o conhecimento tácito (Stewart, 2002).

Esse espaço pode ser físico (como uma sala), virtual (como uma intranet) ou mental (como trocas de experiências e opiniões) ou alguma combinação dos mesmos.

Nonaka e Takeuchi (2004, p. 100), indicam ainda que o "ba é um local existencial onde os participantes partilham seu contexto e criam novos significados por meio de interações".

Na sua forma mais complexa, "ba", como mostra a Figura 1, engloba o surgimento de relações dentro do contexto de um espaço compartilhado que possibilita o compartilhamento de conhecimento, especialmente o conhecimento tácito (Chinying, 2001; Nonaka \& Konno, 1998). Além disso, Fayard (2003) salienta que o conceito "ba" serve como o contexto que permite o início do processo de socialização, externalização, combinação e internalização (SECI) do conhecimento coletivo. 


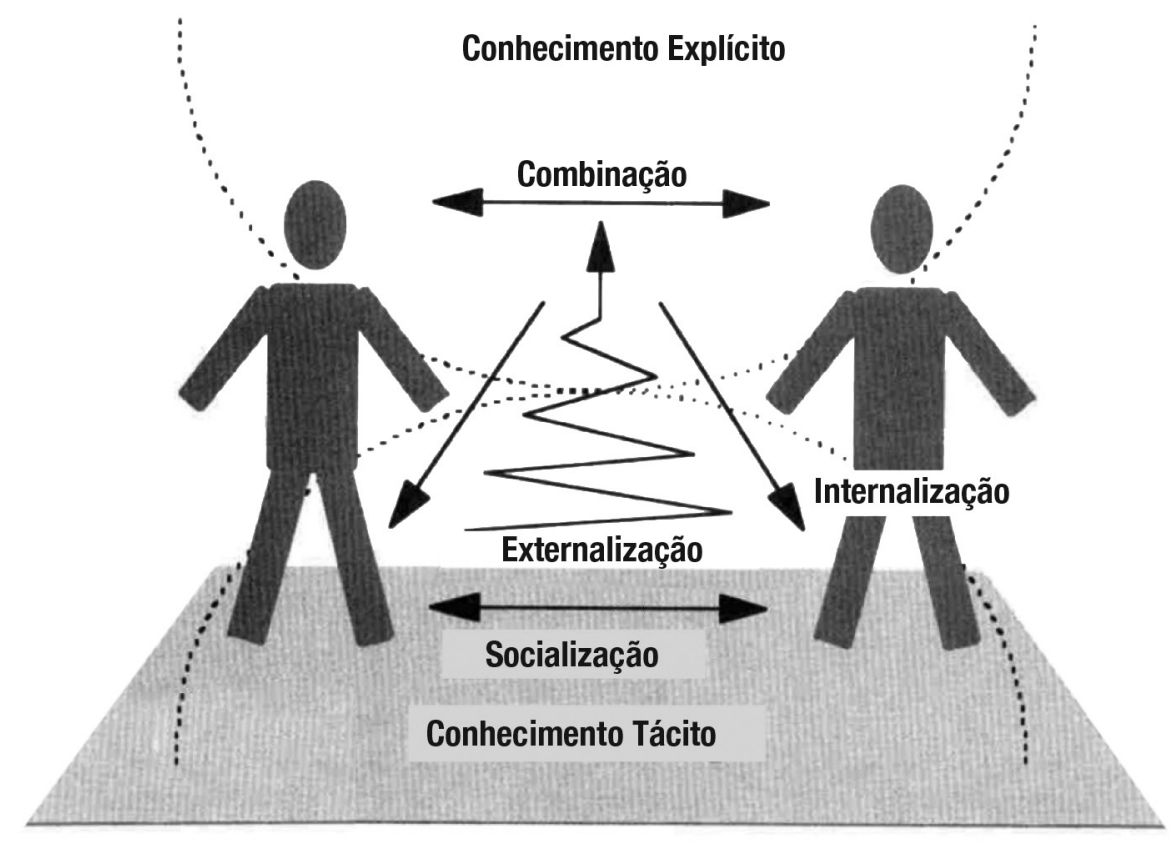

Figura 1. Plataforma Ba

Fonte: Adaptado de Nonaka e Konno (1998, p. 44)

Em essência, o "ba" fornece ao aprendizado organizacional uma plataforma para a existência do um ambiente favorável, uma vez que permite compartilhar, criar e capturar o conhecimento (Vorakulpipat \& Rezgui, 2008).

Nonaka, et al. (2005) apresentam quatro grupos de espaços de criação de conhecimento: espaços de socialização (originating ba), que permitem o envolvimento face a face, o espaço compartilhado, que permitem experiências, emoções e modelos mentais; os espaços de incorporação (exercising ba), que apoiam a internalização do conhecimento explícito e engajamento em exercícios repetitivos por meio do uso de manuais de instrução ou programas de simulação; os espaços de explicitação (dialoguing ba), em que os indivíduos compartilham conhecimento tácito com seus pares por meio do uso do diálogo; os espaços de combinação (systematizing ba), nos quais o há o contexto para a interação no mundo virtual. Seu conteúdo é explícito e pode ser compartilhado com várias pessoas simultaneamente em forma escrita (Nonaka, Toyama, \& Konno, 2000; Nonaka, Von Krogh, \& Voelpel, 2006). Cada grupo suporta um modo particular de conversão de conhecimento no processo SECl. Deste modo, o "ba" engloba mais do que uma interação humana casual ou favorável (Choo \& Alvarenga Neto, 2010).

Quando os ambientes de aprendizagem organizacional são criados com base no conceito de "ba", eles se tornam uma incubadora para o desenvolvimento de um fluxo de conhecimento entre o indivíduo e a organização (Choo \& Alvarenga Neto, 2010; Pan \& Howard, 2010). Da mesma forma, Kodama (2005) salienta que o conhecimento requer um contexto pelo qual a natureza da participação é definida e os participantes podem desenvolver uma nova visão por meio de um processo de aprendizagem compartilhada. 


\section{Procedimentos metodológicos}

Essa é uma pesquisa aplicada, pois colabora para fins práticos (Marconi \& Lakatos, 2007). Quanto à abordagem do problema, trata-se de uma pesquisa qualitativa, com o intuito de compreender o que motiva o compartilhamento de conhecimento tácito, mostrando como ambientes e práticas podem otimizar a aprendizagem organizacional em organizações intensivas em conhecimento. É descritiva, pois se propõem a conhecer e interpretar a realidade sem nela interferir (Churchill \& lacobucci, 2006). Esse tipo de pesquisa utiliza, como forma de estudo, técnicas que contemplam a interpretação para poder descrever e traduzir todos os elementos que compõem os dados pesquisados. Com esse tipo de pesquisa, buscou-se obter dados descritivos sobre o a temática em questão, a partir dos dados coletados com os entrevistados. Como procedimento técnico, utilizou-se um estudo multicaso, que segundo Triviños (1987), refere-se a um estudo de caso aplicado em mais de uma população.

O universo de pesquisa foi o de três organizações de radiologia e diagnóstico por imagem de Santa Catarina. A imagiologia médica realizada nas três organizações varia entre exames de rotina até os altamente especializados. Tais organizações são identificadas nesse artigo como O1 (Organização 1), O2 (Organização 2) e O3 (Organização 3). Especificamente, foram pesquisados três pequenos grupos de radiologistas pertencentes a essas organizações. Para selecionar as organizações participantes da pesquisa, considerou-se como primeiro critério possuir pelo menos dez anos de atividade e fazer uso do sistema de comunicação Picture Archiving and Communication System (PACS), que consiste em um sistema de comunicação e arquivamento de imagens.

Os sujeitos da pesquisa foram 43 médicos com especialização em radiologia e diagnóstico por imagem. Justifica-se esta escolha pelo fato desses atores realizarem uma atividade intensiva em conhecimento - o diagnóstico por imagem, além da característica dos grupos de realizarem suas atividades em colaboração no mesmo ambiente físico, diferente de outros profissionais da saúde, em que prevalece a gestão tradicional, que não é foco dessa pesquisa.

A pesquisa foi aplicada entre os meses de junho de 2016 a janeiro de 2017, conforme a disponibilidade dos entrevistados. Inicialmente, foram realizadas observações por um período de 15 (quinze) dias em cada um dos três grupos participantes, com o objetivo de compreender como os atores compartilham conhecimento em seu ambiente natural de trabalho.

Na sequência, foram realizadas entrevistas com o auxílio de um roteiro semiestruturado, organizado a partir do referencial teórico. Os principais assuntos do roteiro abordaram questões relativas às percepções sobre: a) ambientes que motivam o compartilhamento entre os membros do grupo; b) fatores individuais, sociais, organizacionais e do conhecimento que favorecem o comportamento voltado ao compartilhamento do conhecimento; c) principais práticas que favorecem o compartilhamento e a aprendizagem. Realizaram-se 43 (quarenta e três) entrevistas, sendo 21 (vinte e uma) com o primeiro grupo, 12 (doze) com o segundo grupo e mais 10 (dez) com o terceiro grupo nas três organizações estudadas. Essas entrevistas foram gravadas e transcritas de forma integral. 
Para diminuir a intersubjetividade de entendimento dos autores dessa pesquisa, foi realizada uma etapa final de verificação, por meio de um questionário que apresentou os resultados e os transformou em afirmativas fechadas. Em cada afirmativa, o radiologista tinha uma escala Likert para assinalar o quanto concordava com a afirmativa em questão (de 'discordo totalmente' até 'concordo totalmente'). Optou-se por utilizar uma escala ímpar Likert-5, para evitar o viés da não existência de ponto médio, conforme indicam algumas pesquisas relevantes (Darroch, 2005; Inkinen, Kianto, \& Vanhala, 2015).

Os dados coletados durante a investigação e as entrevistas foram analisados por meio da análise temática (Bardin, 2002). Essa análise permitiu descobrir os pontos comuns dos dados coletados. Para analisar a verificação dos resultados utilizou-se a moda, por ser esta uma medida de posição apropriada para pesquisas qualitativas, pois permite sintetizar, em apenas uma informação, a característica desse conjunto de dados.

\section{Apresentação e análise dos motivadores ao compartilhamento de conhecimento tácito}

\section{Categorias e motivadores ao compartilhamento do conhecimento tácito}

Com base nas observações e nas entrevistas realizadas, na Figura 2, apresentam-se as categorias identificadas e os motivadores relacionados. Essas categorias são separadas entre motivações intrínsecas, composta exclusivamente de fatores individuais de motivação e extrínsecas, separada em organizacionais, sociais e do conhecimento, conforme sugerido por Benabou e Tirole (2003). Também são apresentados os estímulos para a ocorrência do comportamento de compartilhamento do conhecimento, tais como: base de conhecimento, experiência, cultura organizacional e tamanho do grupo.

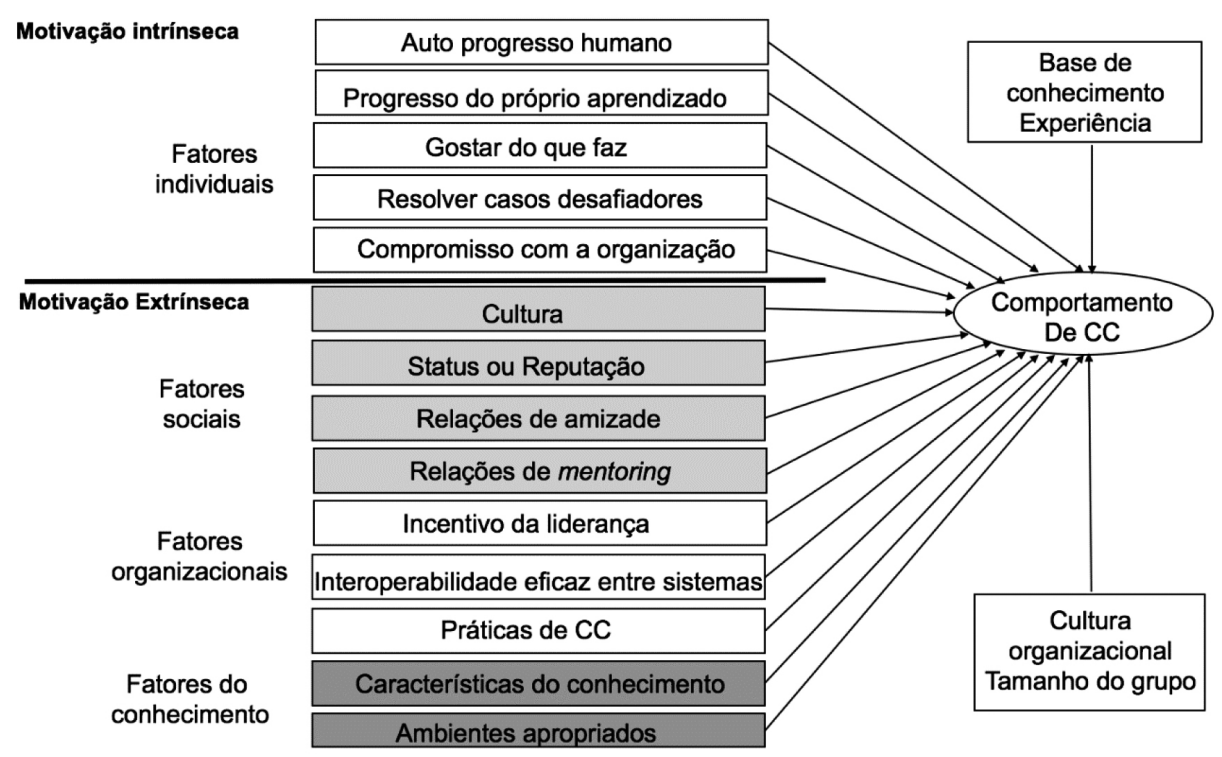

Figura 2. Representação das categorias de motivadores ao compartilhamento do conhecimento

Fonte: Os autores (2017) 
Em relação às motivações para compartilhar conhecimento tácito, foram evidenciados dois achados: i) a percepção de que ao compartilharem conhecimento os radiologistas se beneficiam, por exemplo, da aquisição de novos conhecimentos ou a demonstração de sua competência perante seus pares, o que permite que sejam reconhecidos e admirados; ii) o desencadeamento de muitas outras interações, decorrentes do primeiro episódio de compartilhamento, que permite outros radiologistas a participarem de novas interações. Sendo assim, os radiologistas percebem que um problema ativador de compartilhamento do conhecimento não representa um processo fechado, com início e fim, mas como um processo aberto e contínuo, que pode contribuir para discussões importantes. Isso sugere que, na mente de quem compartilha, uma interação não é uma ação singular e isolada entre destinatário e receptor, mas uma combinação de uma ou mais interações que podem levar a amplos entendimentos.

Conforme Ipê (2003), os fatores que se articulam e atuam no compartilhamento do conhecimento de forma não linear são quatro: i) a natureza do conhecimento; ii) a motivação para compartilhar (que trata o presente artigo); iii) as oportunidades para compartilhar (práticas); e iv) a cultura do ambiente de trabalho.

\section{Análise dos motivadores ao compartilhamento do conhecimento tácito}

Em relação à Motivação intrínseca - fatores individuais, foram identificados cinco motivadores ao compartilhamento do conhecimento: auto progresso humano, progresso do próprio aprendizado, gostar do que faz, resolver casos desafiadores e compromisso com a organização. Essa categoria está relacionada a um sentimento do indivíduo: a satisfação em compartilhar, não necessitando do incentivo de outros atores (Gagné \& Deci, 2005). Assim, permite a criação e o compartilhamento de conhecimento tácito.

O primeiro motivador "auto progresso humano" está ligado à percepção do radiologista de que o compartilhamento do conhecimento ajudará outros colegas, fará com que o mesmo se torne um ser humano melhor e se sentirá bem consigo próprio. Essa compreensão por parte do indivíduo faz com que este se sinta um profissional melhor e seja motivado por esse sentimento. É importante salientar que esse motivador não tem relação com a reciprocidade, pois envolve a ajuda aos colegas sem a expectativa de troca.

Já o motivador "progresso do próprio aprendizado" envolve a percepção de que ao compartilhar suas competências cognitivas com o grupo, o radiologista estará não só ensinando outro indivíduo, mas também aumentando sua base de conhecimento. Os radiologistas comentaram que esse motivador, além de permitir que os participantes sejam mais propensos a compartilhar conhecimento, devido a identificação da oportunidade de melhoria de seu próprio conhecimento, também é uma forma de identificar lacunas, tanto no conhecimento do indivíduo quanto do grupo. Julgase que quando o radiologista se sente mais competente em diagnosticar imagens, passa a se dedicar mais e contribuir de forma mais ativa durante as discussões. 
O motivador "gostar do que faz" foi considerado essencial por boa parte dos entrevistados, não só para compartilhar conhecimento, mas também para trabalhar com diagnóstico por imagem. Os entrevistados afirmavam ainda que, quanto maior a habilidade de o radiologista realizar o diagnóstico e o grau de dificuldade do mesmo, havia mais gosto em resolvê-lo.

Em relação a "resolver casos desafiadores", os entrevistados percebem que quando o diagnóstico é mais complexo, compartilhar conhecimento atrai mais valor. Os casos desafiadores também permitem que o radiologista perceba a sua necessidade de aprendizagem e seja motivado ainda mais a compartilhar conhecimento.

O motivador "compromisso com a organização" consiste em um sentimento de auto melhoramento para melhorar a organização e foi identificado nos três grupos. O fato de estarem comprometidos e se importarem com o nome da organização, não só motiva o compartilhamento para a confirmação da conclusão diagnóstica entre o grupo, como também as interações com radiologistas externos ou outras organizações.

Aa segunda categoria Motivação extrínseca - fatores sociais, refere-se ao encorajamento ou à influência do fator cultural externo e consiste em quatro motivações, manifestadas por meio de interações com outros indivíduos: cultura, status ou reputação, relações de amizade e relações de mentoring.

A "cultura" da organização foi um motivador citado por muitos participantes. De forma semelhante, os três grupos relataram possuir uma atitude positiva em relação ao compartilhamento do conhecimento, formal ou informal. Essa atitude, em grande parte, é decorrência da cultura de trabalho e do clima saudável de convivência existente entre os grupos. O colega não é visto como um competidor ou uma ameaça e sim como parte do todo (o radiologista se vê inserido e cada um possui uma competência importante para o bom funcionamento do todo, da organização). Acreditase que a cultura motiva o compartilhamento, pois os radiologistas possuem normas e valores comuns, sendo assim, consideram trocar conhecimentos como algo natural de seu cotidiano de trabalho.

O motivador "status ou reputação" envolve a necessidade de reconhecimento e encoraja o comportamento favorável ao compartilhamento. Para que sejam percebidos como detentores de conhecimento em seu grupo de trabalho, demonstram o que sabem por meio da explicitação de seu conhecimento.

Apesar de algumas pesquisas não terem conseguido validar a influência positiva da reputação no compartilhamento do conhecimento (Chen, Zhang, Vogel, \& Zhao, 2009; Cho \& Alvarenga Neto, 2010), segundo a percepção dos participantes da presente pesquisa, a reputação motiva sim o compartilhamento do conhecimento. Além disso, a maioria dos participantes indicou que em seus grupos de trabalho o reconhecimento e a visibilidade de seu sucesso são atributos altamente desejáveis. Desse modo, o compartilhamento do conhecimento tácito é motivado por uma cultura de reconhecimento que envolve os relacionamentos afetivos e a confiança.

O motivador "relações de amizade", segundo os entrevistados, está diretamente relacionado a trabalharem lado a lado. Essa proximidade, aliada à convivência constante, promove o compartilhamento de experiências, 
sentimentos e problemas familiares, auxiliando na criação de valores compartilhados. Em relação a tal aspecto, percebe-se que os radiologistas estabelecem entendimentos comuns à medida que trabalham juntos na resolução de diagnósticos críticos. Isso confirma os resultados da pesquisa de Landman, Spatz, Cherlin, Krumholz, Bradley e Curry (2013), que avaliou hospitais de alto desempenho e identificou que os mesmos contavam com estratégias que enfatizavam a comunicação horizontal e a colaboração para melhorar a qualidade do tratamento. As relações de amizade entre os membros emergem, então, da união, cooperação e confiança existente, o que torna o compartilhamento do conhecimento algo natural, corroborando o estudo de Wang e Noe (2010), ao afirmar que, quanto maior o tempo e maior a coesão, mais propensos são os membros da equipe em compartilhar conhecimento.

As "relações de mentoring" são voluntárias e ocorrem em casos específicos, apesar de terem sido relatadas por alguns entrevistados como um forte motivador. Mesmo confirmada uma intensidade acentuada de compartilhamentos motivados por relações de mentoria, essas só foram identificadas nas organizações 1 e 2, e em quatro duplas de radiologistas. O estudo de Joseph e Jacob (2011) demonstra a importância da prática de mentoring e indica ainda a relevância da criação de um programa de tutoria, pois facilita o compartilhamento do conhecimento, principalmente dos recém-chegados para que não se sintam distanciados da comunidade de conhecimento estabelecida.

A terceira categoria, Motivação extrínseca - fatores organizacionais, referente ao relacionamento entre o radiologista e a organização na qual trabalha, identificou três motivadores: incentivo daliderança, interoperabilidade eficaz entre sistemas e práticas de gestão do conhecimento.

Muitos participantes percebem como um diferencial ao compartilhamento, o "incentivo da liderança". Algumas das ações realizadas pelas lideranças para favorecer o compartilhamento, percebidas pelos radiologistas, são: incentivar os radiologistas a fazerem parte de fóruns virtuais para discussão (nas três organizações); provocar discussões entre o grupo nos encontros formais (O1 e O3); conscientizar os radiologistas da importância de esclarecer incoerências encontradas na revisão por pares (01 e O3); supervisionar e orientar os mais experientes a fornecerem feedback aos menos experientes durante a rotina de trabalho (nas três organizações).

O motivador "interoperabilidade eficaz entre sistemas" está ligado à velocidade e qualidade de integração entre a comunicação dos sistemas. Dessa forma, evidenciou-se que a tecnologia não só diminui custos, como também aumenta a eficiência do compartilhamento e possibilita feedback e interações instantâneas, que podem possibilitar o aprendizado com qualquer radiologista que tenha acesso a esse sistema de comunicação.

Algumas "práticas" também motivam o compartilhamento. Como os radiologistas são os principais fomentadores do compartilhamento, as práticas de compartilhamento de conhecimento apropriadas à cultura organizacional e bem suportadas pelos processos, pela tecnologia e pela liderança, podem potencializar o compartilhamento, conforme foi explicitado pelos participantes. As práticas de socialização são importantes, pois permitem que os radiologistas tenham um período de tempo exclusivo durante o trabalho para compartilhar e criar conhecimento. 
Finalmente, na quarta categoria, Motivação extrínseca - fatores do conhecimento, que envolve as caraterísticas do conhecimento compartilhado entre os radiologistas, foram identificados dois motivadores: características do conhecimento e ambientes apropriados.

O primeiro motivador é relacionado às "características do conhecimento", decorrentes da necessidade e dos interesses comuns sobre o conhecimento dominado por determinado subgrupo de trabalho, como a neurologia. A maior intensidade de compartilhamentos entre subgrupos pertencentes ao mesmo domínio foi evidenciada principalmente nas organizações 1 e 2. Na organização 3 não existem subgrupos de trabalho, então, o compartilhamento é mais homogêneo, apesar de os radiologistas pertencentes a essa organização também relatarem uma maior motivação em compartilhar conhecimento com colegas mais experientes.

O motivador "ambientes apropriados" envolve os diferentes tipos de ambientes disponíveis para interações físicas e/ou virtuais. Os radiologistas percebem que as interações ocorridas na sala de laudos são mais ricas devido ao tamanho do grupo. Além disso, tais interações são mais valiosas por envolverem não só a comunicação face a face em si, como também a observação em conjunto das imagens e a compreensão de outras emoções que podem ser causadas por medo de errar, problemas familiares, excesso de trabalho e falta de tempo.

\section{Contribuições para o avanço da teoria de compartilhamento de conhecimento tácito}

O layout de trabalho aberto da sala foi comentado pela maioria dos participantes. Nas modalidades diagnósticas nas quais os radiologistas não realizavam seu trabalho na sala de laudo, como mamografia e ultrassom, o comportamento de ajuda era menor entre os membros. Apesar de os mesmos se encontrarem de maneira formal (organização 1 e 3), possuíam pouca familiaridade e compartilhavam menos com o grande grupo. Ademais, afirmaram gostar dessa forma de trabalhar, sem portas ou paredes.

Embora o ambiente da sala de laudos fosse mais barulhento (e alguns participantes comentaram sobre isso), ninguém faria mudanças quanto ao fato de manter o grupo junto no mesmo espaço. Outros tipos de ambientes, porém virtuais, que motivaram o compartilhamento, foram o PACS e o Mobile Messenger. O PACS consiste em um sistema que possibilita melhorar o desempenho do diagnóstico dos radiologistas, além de permitir representar o conhecimento abstrato, de alto nível, sobre imagens e compartilhar opiniões durante o diagnóstico mesmo em locais separados geograficamente (Zhou, Liu, \& Le, 2007).

No contexto dessa pesquisa, a riqueza do ambiente não está relacionada somente à diversidade de canais disponíveis para compartilhar, mas também a sua aplicação efetiva por parte dos radiologistas. Pode-se perceber que existem diferentes fatores que motivam os radiologistas a compartilhar conhecimento. Porém, nos três grupos estudados, foi percebida uma forte cultura de compartilhamento do conhecimento, fruto de relações de confiança, afetividade e cooperação. Os valores comuns que os radiologistas 
possuem fornecem um modelo de confiança que faz com que os indivíduos do grupo se empenhem para desenvolver suas relações com os outros. Trabalhar na mesma sala permite conhecer a reputação, as emoções atuais e o ânimo dos membros, o que pode definir a escolha de quem compartilhar. Em consequência do crescimento desses sentimentos, os radiologistas se mostraram mais motivados a compartilhar conhecimento, principalmente tácito. Esse resultado confirma o estudo de Yang e Farn (2009), ao abordar que a intenção de compartilhar conhecimento tácito é afetada pela confiança baseada nos afetos e valores compartilhados.

Conforme já abordado nos procedimentos metodológicos, foi realizada uma verificação dos resultados com os participantes. Tais resultados mostram que houve ampla concordância entre os participantes em relação à devolução das afirmativas, que sintetizaram os resultados percebidos pelos autores dessa pesquisa. Somente nas afirmativas que estavam relacionadas às práticas de mentoring, encontros de grupo e a revisão por pares, alguns participantes assinalaram a opção "discordo totalmente". Essas discordâncias são explicadas pelo fato de que nem todas as três organizações pesquisadas possuem essas práticas instituídas em sua rotina organizacional.

Em relação às afirmativas que tiveram a maior ocorrência de "concordo totalmente", destacam-se, respectivamente, aquelas que configuram a percepção do radiologista de que: a) ao ensinar aos colegas também se aprende; b) gostar da atividade amplia a motivação para compartilhar conhecimento; e c) o compartilhamento do conhecimento ajuda a melhorar a qualidade dos diagnósticos e, consequentemente, a imagem da organização e do profissional.

O resultado obtido na verificação dos resultados reforça e confirma os entendimentos apresentados nessa pesquisa em relação a importância da motivação para efetivar o compartilhamento do conhecimento, principalmente quando essa motivação está relacionada à oportunidade de aprendizagem percebida nas interações, a realmente gostar da atividade realizada e ao reconhecimento do indivíduo pelo conhecimento que possui.

Este estudo descobriu que os radiologistas tinham i) a percepção de que ao compartilharem conhecimento também se beneficiam, por exemplo, da aquisição de novos conhecimentos como "progresso do próprio aprendizado" ou a demonstração de sua competência perante seus pares, o que permite que sejam reconhecidos e admirados; ii) o desencadeamento de muitas outras interações, decorrentes do primeiro episódio de compartilhamento, o que permite a outros radiologistas participar de novas interações.

Além disso, o estudo confirmou que "resolver casos desafiadores" motivou o compartilhamento de conhecimento tácito, pois os entrevistados se mostraram mais interessados em compartilhar quando o diagnóstico era mais complexo e enfatizaram que nesses casos compartilhar conhecimento atrai mais valor.

Apesar de algumas pesquisas não terem conseguido validar a influência positiva da reputação no compartilhamento do conhecimento (Chen, Zhang, Vogel, \& Zhao, 2009; Cho \& Alvarenga Neto, 2010), segundo a percepção dos participantes da presente pesquisa, a reputação motiva 
sim o compartilhamento do conhecimento. Além disso, a maioria dos participantes indicou que em seus grupos de trabalho, o reconhecimento e a visibilidade de seu sucesso são atributos altamente desejáveis. Desse modo, o compartilhamento do conhecimento tácito é motivado pelo desejo de ser reconhecido perante o grupo.

Os resultados desta pesquisa confirmaram que existia uma diferença significativa quanto à motivação para compartilhar conhecimento tácito quando o conhecimento a ser compartilhado era de uma área específica de atuação do indivíduo (motivador características do conhecimento), como o conhecimento relacionado a neurologia. Nesses casos, o compartilhamento era de grande interesse do indivíduo neurologista.

Conforme apresentado ao longo do trabalho, o tamanho dos grupos e as características peculiares dos participantes de cada grupo com certeza exerceram influência quanto ao motivador "relações de amizade", que permitiu a criação de confiança existente nos grupos. Essas relações, por sua vez, facilitaram o efetivo desenvolvimento de algumas das práticas de aprendizagem, como a revisão por pares, o storyteling e o mentoring.

Os entrevistados mostraram intenção em compartilhar seus conhecimentos tácitos motivados pela "cultura de trabalho", na qual o colega não é visto como um competidor ou uma ameaça, mas como parte do todo (o radiologista se vê inserido no grupo e cada um possui uma competência importante para o bom funcionamento do todo, da organização). A cultura motiva o compartilhamento, pois os integrantes da organização possuem a percepção do contexto favorável ao compartilhamento por meio das normas e valores comuns, sendo assim, consideram trocar conhecimentos como algo natural de seu cotidiano de trabalho.

A implicação das descobertas realizadas na pesquisa de campo para a teoria sobre compartilhamento de conhecimento tácito permite avançar no conhecimento existente de modo que apresenta novas descobertas em relação a essa temática.

Destaca-se que as contribuições desta pesquisa para o campo de conhecimentos são: a importância do indivíduo ser reconhecido para poder compartilhar seu conhecimento tácito; a percepção do indivíduo de que ele aprende ao compartilhar conhecimento tácito; a maior motivação no compartilhamento dos casos que são desafiadores e do conhecimento que é específico ao domínio do indivíduo; a maior efetividade de algumas práticas como revisão por pares, storyteling e mentoring, devido às características peculiares do grupos e da percepção que os mesmos possuem da ação de compartilhar.

Outrossim, foi verificado que quando o indivíduo gosta da atividade que realiza e é reconhecido pelo seu grupo de trabalho pelo conhecimento que possui, sua motivação para compartilhar conhecimento é ampliada, além de perceber o compartilhamento do conhecimento como uma oportunidade de aprendizado. 


\section{Considerações finais e recomendações}

O objetivo geral dessa pesquisa foi evidenciar os motivadores ao compartilhamento de conhecimento tácito em três organizações de radiologia e diagnóstico por imagem, consideradas intensivas em conhecimento.

Foi possível observar que há um ponto de vista comum entre os radiologistas no que se refere ao entendimento dos elementos motivadores, quando se trata do comportamento individual e do grupo.

Como principal resultado, o tema motivação foi classificado em motivação intrínseca e motivação extrínseca, caracterizando quatro categorias de motivação relacionadas a: i) fatores individuais, ii) fatores sociais, iii) fatores organizacionais e iv) fatores do conhecimento. Esses fatores atuam no comportamento de compartilhamento do conhecimento, juntamente com a base de conhecimento e experiência do indivíduo, cultura organizacional e tamanho do grupo de trabalho.

Inicialmente, percebe-se nos "fatores individuais" que gostar da atividade surge como principal motivador no discurso de todos os participantes. Sendo assim, esses resultados confirmam a pesquisa de McDermott e O'Dell (2001) que indicam que o motivador mais significativo ao compartilhamento está ligado às recompensas intrínsecas vindas a partir do próprio trabalho ou por um sentimento de envolvimento e contribuição com o grupo.

Os "fatores do conhecimento", que representam o ambiente apropriado revelaram estimular a necessidade de otimizar o aprendizado organizacional "ba" e o compartilhamento de conhecimento tácito dentro da organização. Dessa forma, os contextos de trabalho estudados ajudavam na criação de relacionamentos entre os radiologistas que alavancam as possibilidades e a capacidade dos atores de aprenderem a compartilhar e adquirir conhecimento. Isso confirma a pesquisa de Tonet e Paz (2006), ao relatar que o contexto organizacional em que a fonte está inserida pode influenciar de forma positiva ou negativa o compartilhamento do conhecimento.

Em relação aos "fatores sociais", o reconhecimento foi citado pelos entrevistados como um motivador para compartilhar principalmente o conhecimento tácito. Quanto aos "fatores organizacionais", o incentivo da liderança e as práticas de compartilhamento do conhecimento destacaram-se.

Fundamenta-se outro ponto de inferência acerca dos resultados encontrados, levando em consideração que as relações de amizade entre os radiologistas em cada um dos três grupos se mostraram imprescindíveis para que ocorresse o compartilhamento de conhecimento tácito. Como consequência, há criação de confiança que tende a aumentar ainda mais o comportamento para compartilhar aquele conhecimento que é crítico. Essa afetividade, aliada ao indivíduo ser reconhecido pelo conhecimento que possui, são vitais para que o compartilhamento de conhecimento tácito não seja percebido pelo compartilhador como uma possível perda de poder.

As principais considerações dessa pesquisa enfatizam o valor das relações afetivas e foram consistentes com a visão de Whittaker e Van 
Beveren (2005, p. 119), que argumentam que o compartilhamento eficaz de conhecimento nos cuidados de saúde exige a construção em conjunto para envolver múltiplas perspectivas. Os autores ainda complementam que "o capital social existe em uma relação entre dois atores se eles desenvolvem vínculos pessoais, apego e confiança".

Assim, o fator que auxilia o processo de compartilhamento do conhecimento tácito, conforme sugerido por essa pesquisa e pesquisas evidenciadas no referencial teórico, está relacionado diretamente ao envolvimento face a face. Isto implica em considerar que os esforços estratégicos para aumentar a proximidade entre os membros do grupo devem priorizar a realização conjunta do trabalho em ambientes abertos. Esses fatores minimizam um problema comum que pode ser um inibidor ao compartilhamento, ou seja, a distância entre os membros. Além disso, alguns grupos estudados realizam, periodicamente, encontros destinados à interação entre os membros com o intuito de discutir problemas que são críticos à atividade de diagnóstico por imagem. O comprometimento e o engajamento dos atores foram motivados ainda pela liderança que estrutura e organiza a realização de práticas como a revisão por pares.

Os resultados desta pesquisa são consistentes com o estudo de Landman et al. (2013) e Leever et al. (2010) ao mencionarem que os médicos que possuem respeito mútuo, confiança, profissionalismo e percebem a competência dos seus colegas, são mais dispostos a estabelecer contato e assegurar uma compreensão comum com outros profissionais da saúde.

O suporte oferecido pela tecnologia foi reconhecido por melhorar o desempenho ao aumentar a produtividade do compartilhamento de conhecimento. O PACS e o Mobile Messenger desempenham um papel crítico na criação, armazenamento e distribuição de conhecimento explícito de forma acessível e rápida. A facilidade do uso da tecnologia utilizada pelos indivíduos foi uma característica que favoreceu seu uso comum.

Sendo assim, o rodízio inevitável dos trabalhadores do conhecimento implica que as informações obtidas em um ambiente irão inevitavelmente ultrapassar as fronteiras organizacionais. Isso indica que as organizações continuarão ase beneficiar da criação de redes deaprendizagem organizacional para permitir a otimização do compartilhamento de conhecimento tácito, minimizando as perdas de tal conhecimento.

Este estudo contribuiu para a literatura de compartilhamento de conhecimento tácito, ao observar que alguns fatores motivacionais foram contraditórios a literatura utilizada (Chen, Zhang, Vogel, \& Zhao, 2009; Cho \& Alvarenga Neto, 2010). Um exemplo disso é a identificação do reconhecimento como um motivador ao compartilhamento de conhecimento tácito.

Essas descobertas fornecem grandes contribuições para as organizações, que ao invés de apenas gastarem bilhões de dólares em software de gerenciamento do conhecimento podem também fazer uso de diferentes abordagens para motivar e incentivar os indivíduos a se sentirem parte do grupo, só assim, o conhecimento tácito inerente aos indivíduos será compartilhado por todo grupo contribuindo para a vantagem competitiva da organização. 
Por fim, evidencia-se que as considerações desta pesquisa são exclusivas para os ambientes estudados. Dessa forma, a partir destas ponderações e com o objetivo de aumentar o entendimento do assunto para a área da saúde, sugere-se que outros estudos utilizem o que foi explicitado nessa análise para realizar novos estudos com diferentes populações, amostras e fatores motivacionais, de modo a ampliar a criação de novos entendimentos referentes ao tema.

\section{Referências}

Aizpurúa, L. I., Saldaña, P. E. Z., \& Saldaña, A. Z. (2011). Learning for sharing: an empirical analysis of organizational learning and knowledge sharing. International Entrepreneurship and Management Journal, 7(4), 509-518.

Al-Hawamdeh, S. (2002). Knowledge management: re-thinking information management and facing the challenge of managing tacit knowledge. Information Research, 8(1), 8-1.

Alavi, M., \& Leidner, D. E. (2001). Review: Knowledge management and knowledge management systems: Conceptual foundations and research issues. MIS Quarterly 25(1), 107-136.

Alvesson, M. (2004). Knowledge work and knowledge-intensive firms. Oxford; New York: Oxford University Press.

Bardin, L. (2002). Análise de conteúdo. Lisboa: Edições 70.

Bejinaru, R., and lordache, S. (2010). Knowledge channeling in the learning organization. In Proceedings of the 5th International Conference on Business Excellence (pp. 59-62). Brasov: Infomarket Publishing House.

Benabou, R., \& Tirole, J. (2003). Intrinsic and extrinsic motivation. The Review of Economic Studies, 70(3), 489-520.

Benson, J. \& P. Debroux. (2013) Changing Nature of Management and Culture in Japan, In: M. Warner (ed), Managing across diverse cultures in East Asia, Routledge.

Borges, M. D. L. (2012). Produção de sentido em processos decisórios: um estudo no contexto de eventos inesperados na perspectiva da análise da conversa, Tese de Doutorado, Universidade do Vale do Rio dos Sinos, São Leopoldo, RS, Brasil.

Celadon, K. (2005). O compartilhamento do conhecimento no âmbito intraempresarial: um estudo de caso. Dissertação de Mestrado, Universidade Tecnológica Federal do Paraná, Curitiba, PR, Brasil.

Chen, Z., Vogel, D., Zhang, X., \& Zhao, D. (2009). Encouraging knowledge sharing in global virtual teams: The interaction effect of individual difference and perceived sharing benefits. From the Proceedings of the 42nd Hawaii International Conference on System Sciences (1-10). Waikoloa, HI.

Choi, B., \& Lee, H. (2002). Knowledge management strategy and its link to knowledge creation process. Expert Systems with Applications, 23(3), 173-187.

Choo, C. W. and Alvarenga Neto, R. (2010). Beyond the ba: managing enabling contexts in knowledge organizations, Journal of Knowledge Management, 14(4), 592-610. 
Churchill, G. A., \& lacobucci, D. (2006). Marketing research: methodological foundations. New York: Dryden Press.

Collins, H., \& Evans, R. (2010). Repensando a expertise. Belo Horizonte: Fabrefactum.

Dabbagh, N., \& Kitsantas, A. (2012). Personal Learning Environments, social media, and self-regulated learning: A natural formula for connecting formal and informal learning. The Internet and higher education, 15(1), 3-8.

Darroch, J. (2005). Knowledge management, innovation and firm performance. Journal of Knowledge Management, 9(3), 101-115.

Davenport, T. H., \& Prusak, L. (1998). Working knowledge: how organizations manage what they know. Harvard Business School Press, Boston.

De Long, D. W., \& Fahey, L. (2000). Diagnosing cultural barriers to knowledge management. Academy of Management Perspectives, 14(4), 113-127.

Detienne, K., Dyer, G., Hoopes, C., \& Harris, S. (2004). Toward a model of effective knowledge management and directions for future research: Culture, leadership, and CKOs. Journal of leadership \& organizational studies, 10(4), 26-43.

Dixon, N. (2002). The neglected receiver. Ivey Business Journal, 66(4), 35-40.

Fayard, P. M. (2003). Strategic communities for knowledge creation: A Western proposal for the Japanese concept of Ba. Journal of Knowledge Management, 7(5), 25-31.

Gagné, M. (2009). A model of knowledge sharing motivation. Human Resource Management, 48(4), 571-589.

Gagné, M., \& Deci, E. L. (2005). Self determination theory and work motivation. Journal of Organizational Behavior, 26(4), 331-362.

Goulart, E., \& Angeloni, M. T. (2009). O compartilhamento do conhecimento em uma instituição de ensino superior. Revista Brasileira de Docência, Ensino e Pesquisa em Administração, Edição Especial, 1(2), 59-83.

Grant, K. A. (2007). Tacit knowledge revisited-we can still learn from Polanyi. The Electronic Journal of Knowledge Management, 5(2), 173-180.

Greenberg, J., \& Baron, R. A. (1997). Behavior in organizations: understanding and managing the human side of work (6th. ed.). Upper Saddle River, NJ: Prentice Hall.

Gupta, A. K., \& Govindarajan, V. (2000). Knowledge flows within multinational corporations. Strategic management journal, 21(4), 473-496.

Hildreth, P. M., \& Kimble, C. (2002). The duality of knowledge. Information Research $8(1)$.

Hildreth, P., Kimble, C., \& Wright, P. (2000). Communities of practice in the distributed international environment. Journal of Knowledge Management, 4(1), 27-38.

Hunter, L., Beaumont, P., \& Lee, M. (2002). Knowledge management practice in Scottish law firms. Human Resource Management Journal, 12(2), 4-21.

Husted, K., \& Michailova, S. (2002). Diagnosing and fighting knowledge-sharing hostility. Organizational Dynamics, 31(1), 60-73.

Inkinen, H. T., Kianto, A., \& Vanhala, M. (2015). Knowledge management practices and innovation performance in Finland. Baltic Journal of Management, 10(4), 432-455. 
Ipê, M. (2003). Knowledge sharing in organizations: A conceptual framework. Human Resource Development Review, 2(4), 337-359.

Işik O., Mertens, W., \& Van Den Bergh, J. (2013) Practices of knowledge intensive process management: quantitative insights. Business Process Management Journal, 19(3), 515-534.

Joseph, B., \& Jacob, M. (2011). Knowledge sharing intentions among IT professionals in India. Communications in Computer and Information Science, 114, 23-31.

Khasawneh, S. (2011). Shared leadership and organizational citizenship behaviour in Jordanian public universities: Developing a global workforce for the 21st Century. Educational Management Administration \& Leadership, 39(5), 621-634.

Kim, S., \& Lee, H. (2006). The impact of organizational context and information technology on employee knowledge-sharing capabilities. Public Administration Review, 66(3), 370-385.

Kodama, M. (2005). New knowledge creation through leadership-based strategic community - a case of new product development in IT and multimedia business fields. Technovation, 25(8), 895-908.

Kumaraswamy, K.S.N., Chitale, C.M., 2012. Collaborative knowledge sharing strategy to enhance organizational learning. Journal of Management Development, 31(3), 308-322.

Landman, A. B., Spatz, E. S., Cherlin, E. J., Krumholz, H. M., Bradley, E. H., \& Curry, L. A. (2013). Hospital collaboration with emergency medical services in the care of patients with acute myocardial infarction: perspectives from key hospital staff. Annals of Emergency Medicine, 61(2), 185-195.

Lang, J. (2001). Managerial concerns in knowledge management. Journal of Knowledge Management, 5(1), 43-59.

Latham, G. P., \& Pinder, C. C. (2005). Work motivation theory and research at the dawn of the twenty-first century. Annual Review of Psychology, 56(1), 485-516.

Lee, P., Gillespie, N., Mann, L., \& Wearing, A. (2010). Leadership and trust: Their effect on knowledge sharing and team performance. Management Learning, 41(4), 473-491.

Leever, A. M., Hulst, M. V. D., Berendsen, A. J., Boendemaker, P. M., Roodenburg, J. L. N., \& Pols, J. (2010). Conflicts and conflict management in the collaboration between nurses and physicians-A qualitative study. Journal of Interprofessional Care, 24(6), 612-624.

Lin, H. F., Lee, H. S., \& Wang, D. W. (2009). Evaluation of factors influencing knowledge sharing based on a fuzzy AHP approach. Journal of Information Science, 35(1), 25 44.

Mahmood, A., Qureshi, M. A., \& Shahbaz, Q. (2011). An examination of the quality of tacit knowledge sharing through the theory of reasoned action. Journal of Quality and Technology Management, 7(1), 39-55.

Marconi, M. D. A., \& Lakatos, E. M. (2007). Fundamentos da metodologia. (6 ed.). São Paulo: Atlas.

McDermott, R., \& O'Dell, C. (2001). Overcoming cultural barriers to sharing knowledge. Journal of Knowledge Management, 5(1), 76-85. 
Nomura, T. (2002). Design of 'Ba'for successful knowledge management how enterprises should design the places of interaction to gain competitive advantage. Journal of Network and Computer Applications, 25(4), 263-278.

Nonaka, I., \& Konno, N. (1998). The concept of" ba": Building a foundation for knowledge creation. California Management Review, 40(3), 40-54.

Nonaka, I., \& Takeuchi, H. (1995). The knowledge-creating company: how Japanese companies create the dynamics of innovation. USA: Oxford University Press.

Nonaka, I., \& Takeuchi, H. (1997). Criação de conhecimento na empresa: como as empresas japonesas geram a dinâmica da inovação. Rio de Janeiro: Campus.

Nonaka, I., \& Takeuchi, H. (2004). Criação de conhecimento na empresa. Rio de Janeiro: Elsevier Brasil.

Nonaka, I., \& Von Krogh, G. (2009). Perspective - tacit knowledge and knowledge conversion: controversy and advancement in organizational knowledge creation theory. Organization Science, 20(3), 635-652.

Nonaka, I., Byosiere, P., Borucki, C. C., \& Konno, N. (1994). Organizational knowledge creation theory: a first comprehensive test. International Business Review, 3(4), 337-351.

Nonaka, I., Toyama, R., \& Konno, N. (2000). SECl, Ba and leadership: a unified model of dynamic knowledge creation. Long Range Planning, 33(1), 5-34.

Nonaka, I., Von Krogh, G., \& Voelpel, S. (2006). Organizational knowledge creation theory: Evolutionary paths and future advances. Organization Studies, 27(8), 1179-1208.

Nonaka, S., Yoshiba, S., Watanabe, D., Ikeuchi, S., Goto, T., Marshall, W. F., \& Hamada, H. (2005). De novo formation of left-right asymmetry by posterior tilt of nodal cilia. PLoS Biology, 3(8), 268.

Pan, D., \& Howard, Z. (2010). Distributing leadership and cultivating dialogue with collaborative EBIP. Library Management, 31(7), 494-504.

Polanyi, M. (1966). The tacit dimension. Chicago: The University of Chicago Press.

Senoo, D., Magnier-Watanabe, R., \& Salmador, M. P. (2007). Workplace reformation, active ba and knowledge creation: From a conceptual to a practical framework. European Journal of Innovation Management, 10(3), 296-315.

Servin, G. (2005). ABC of knowledge management. NHS National Library for Health: Knowledge Management Specialist Library, available at http://www.library.nhs.uk/ knowledge management/

Sharkie, R. (2005). Precariousness under the new psychological contract: The effect on trust and the willingness to converse and share knowledge. Knowledge Management Research \& Practice, 3(1), 37-44.

Starbuck, W. H. (1992). Learning by knowledge intensive firms. Journal of management Studies, 29(6), 713-740.

Stenmark, D. (2000). Leveraging tacit organizational knowledge. Journal of management Information Systems, 17(3), 9-24.

Stewart, T. A. (2002). The case against knowledge management. Business, 2(3), 2.

Tonet, H. C., \& Paz, M. D. G. T. D. (2006). Um modelo para o compartilhamento de conhecimento no trabalho. Revista de Administração Contemporânea, 10(2), 75-94. 
Triviños, A. N. S. (1987). Introdução à pesquisa em ciências sociais: a pesquisa qualitativa em educação. São Paulo: Atlas.

Van Den Hooff, B., \& De Ridder, J. A. (2004). Knowledge sharing in context: the influence of organizational commitment, communication climate and $\mathrm{CMC}$ use on knowledge sharing. Journal of Knowledge Management, 8(6), 117-130.

Von Krogh, G., \& Roos, J. (1995). Organizational epistemology. New York: St. Martin's Press.

Von Krogh, G., Ichijo, K., \& Nonaka, I. (2001). Facilitando a criação de conhecimento: reiventando a empresa com o poder da inovação contínua. Rio de Janeiro: Campus.

Von Krogh, G., Nonaka, I., \& Rechsteiner, L. (2012). Leadership in organizational knowledge creation: a review and framework. Journal of Management Studies, 49(1), 240-277.

Vorakulpipat, C., \& Rezgui, Y. (2008). An evolutionary and interpretive perspective to knowledge management. Journal of Knowledge Management, 12(3), 17-34.

Walsham, G. (1993). Interpreting information systems in organizations. New York: John Wiley.

Wang, S., \& Noe, R. A. (2010). Knowledge sharing: A review and directions for future research. Human Resource Management Review, 20(2), 115-131.

Wei, J., Zheng, W., \& Zhang, M. (2011). Social capital and knowledge transfer: A multi-level analysis. Human Relations, 64(11), 1401-1423.

Whittaker, J., \& Van Beveren, J. (2005). An Important Ingredient to Effective Knowledge Sharing: Meditute, A Case Study. Clinical Knowledge Management, 5(297), 83-113.

Yang, S. C., \& Farn, C. K. (2009). Social capital, behavioural control, and tacit knowledge sharing - a multi-informant design. International Journal of Information Management, 29(3), 210-218.

Zhou, Z., Liu, B. J., \& Le, A. H. (2007). CAD-PACS integration tool kit based on DICOM secondary capture, structured report and IHE workflow profiles. Computerized Medical Imaging and Graphics, 31(4), 346-352. 